\title{
LDPC-Coded 16-Dimensional Modulation Based on the Nordstrom-Robinson Nonlinear Block Code
}

\author{
Koike-Akino, T.; Millar, D.S.; Kojima, K.; Parsons, K.; Sugihara, K.; Miyata, Y.; Yoshida, T.
}

TR2015-038 May 2015

\begin{abstract}
Recent forward error correction (FEC) codes based on low-density parity-check (LDPC) codes have shown capacity-achieving performance in practice. To support various modulation formats in conjunction with LDPC codes, bit-interleaved coded modulation (BICM) has been widely used. To achieve better performance, BICM iterative demodulation (BICM-ID), multi-layer coding (MLC), and nonbinary-interleaved coded modulation (NBICM) have been studied for highorder modulation and high-dimensional modulation (HDM). In particular, HDM has received much interest because of its high sensitivity. In this paper, we propose a new 16-dimensional (16D) modulation based on the Nordstrom-Robinson (NR) nonlinear code. The NR code is an optimal binary code, whose minimum Hamming distance is 6, whereas the best-known linear code (BKLC) has a minimum distance of 5. We optimize LDPC codes for BICM-ID with various HDM formats, and show the benefit of the NR-coded 16D modulation.
\end{abstract}

Conference on Lasers and Electro-Optics (CLEO)

This work may not be copied or reproduced in whole or in part for any commercial purpose. Permission to copy in whole or in part without payment of fee is granted for nonprofit educational and research purposes provided that all such whole or partial copies include the following: a notice that such copying is by permission of Mitsubishi Electric Research Laboratories, Inc.; an acknowledgment of the authors and individual contributions to the work; and all applicable portions of the copyright notice. Copying, reproduction, or republishing for any other purpose shall require a license with payment of fee to Mitsubishi Electric Research Laboratories, Inc. All rights reserved. 



\title{
LDPC-Coded 16-Dimensional Modulation Based on the Nordstrom-Robinson Nonlinear Block Code
}

\author{
Toshiaki Koike-Akino ${ }^{1}$, David S. Millar ${ }^{1}$, Keisuke Kojima ${ }^{1}$, Kieran Parsons ${ }^{1}$, \\ Kenya Sugihara ${ }^{2}$, Yoshikuni Miyata ${ }^{2}$, and Tsuyoshi Yoshida ${ }^{2}$ \\ Mitsubishi Electric Research Labs. (MERL), 201 Broadway, Cambridge, MA 02139,USA. koike@merl.com \\ Information Technology R\&D Center, Mitsubishi Electric Corporation, 5-1-1 Ofuna, Kanagawa 247-8501, Japan.
}

\begin{abstract}
We propose a new high-dimensional modulation (HDM) format, based on the Nordstrom-Robinson code, which is the best-known nonlinear block code in 16 dimensions. With EXIT chart, we optimize LDPC codes for various HDM formats, and show their benefits.
\end{abstract}

OCIS codes: (060.4510) Optical communications, (060.1660) Coherent communications, (060.4080) Modulation.

\section{Introduction}

Recent forward error correction (FEC) codes based on low-density parity-check (LDPC) codes [1-6] have shown capacity-achieving performance in practice. To support various modulation formats in conjunction with LDPC codes, bit-interleaved coded modulation (BICM) has been widely used. To achieve better performance, BICM iterative demodulation (BICM-ID), multi-layer coding (MLC), and nonbinary-interleaved coded modulation (NBICM) have been studied for high-order modulation and high-dimensional modulation (HDM). In particular, HDM has received much interest because of its high sensitivity [7-13]. In this paper, we propose a new 16-dimensional (16D) modulation based on the Nordstrom-Robinson (NR) nonlinear code [14-16]. The NR code is an optimal binary code, whose minimum Hamming distance is 6, whereas the best-known linear code (BKLC) [17] has a minimum distance of 5. We optimize LDPC codes for BICM-ID with various HDM formats, and show the benefit of the NR-coded 16D modulation.

\section{LDPC Code Design for High-Dimensional Modulation Based on Block Coding}

HDM provides larger Euclidean distance to be robust against noise. There are many ways to design HDM. In [12], we have proposed to use sphere cutting and lattice packing based on some known densest lattices. This method creates irregular constellations, and has a labeling issue. Alternatively, we can use short block codes to design HDM [11], e.g., single parity-check (SPC), extended Hamming code, extended Golay code, and nonbinary block code for regular quadrature-amplitude modulation (QAM) constellations. Most previous works have focused on linear block codes. When it comes to nonlinear block codes, there exist some known good codes including Preparata codes. In particular, the Nordstrom-Robinson (NR) code [14] achieves the largest minimum Hamming distance for block codes of size 16. Some low-complexity decoding methods are also known for the NR code [16]. In this paper, we consider NRbased HDM and optimize an LDPC code for its BICM-ID application. Note that the NR code is a binary image of a nonbinary $Z_{4}$ code as shown in [15], and the related study for higher-rate HDM formats can be found in [10].

To design LDPC codes for BICM-ID, we use a methodology proposed in [4], where extrinsic information transfer (EXIT) curves of variable-node decoder is combined with that of demodulator. Using this method, we optimize degree distributions of LDPC codes for various HDM formats. The required signal-to-noise ratio (SNR), a.k.a. threshold, of those optimized degree distributions for a code rate of 0.9 is shown in Fig. 1(a), where threshold penalty from Shannon limit is presented as a function of dimensionality for two cases of BICM and BICM-ID. Here, we consider check-concentrated triple-weight LDPC codes, with variable node degrees no larger than 16. It is shown that higher dimensionality improves the threshold. For 8D, 16D, and 24D modulations, BICM-ID outperforms BICM by approximately $2 \mathrm{~dB}$. Note that $16 \mathrm{D}$ modulation with NR code offers approximately $0.2 \mathrm{~dB}$ gain compared to 16D BKLC [17].

\section{BER Performance}

Here, we evaluate bit-error rate (BER) performance of optimized LDPC codes for 8D, 16D, and 24D modulations. Given degree distributions optimized by EXIT chart, we use progressive edge growth (PEG) [18] to achieve larger girth for a codeword length of 38400. We show post-LDPC BER performance of BICM-ID in Fig. 1(b), where we use 32 iterations for turbo demodulation and 1 iteration for BP decoding per turbo loop. We also plot the BICM-ID performance of the LDPC code optimized for 1D modulation, i.e., BICM-optimized code. It is shown that optimized 
LDPC codes for BICM-ID achieve approximately $0.2 \mathrm{~dB}$ gain from the LDPC code optimized for BICM except for $8 \mathrm{D}$ modulation. The new $16 \mathrm{D}$ modulation based on the NR code offers $0.6 \mathrm{~dB}$ gain from $8 \mathrm{D}$ modulation, and $0.1 \mathrm{~dB}$ gain from 16D modulation based on linear code. Moreover, the gap between 16D NR HDM and 24D Golay HDM is only $0.2 \mathrm{~dB}$. Since the computational complexity increases as the dimension increases, the 16D NR HDM may have a superior trade-off between complexity and performance compared to 8D Hamming HDM and 24D Golay HDM.

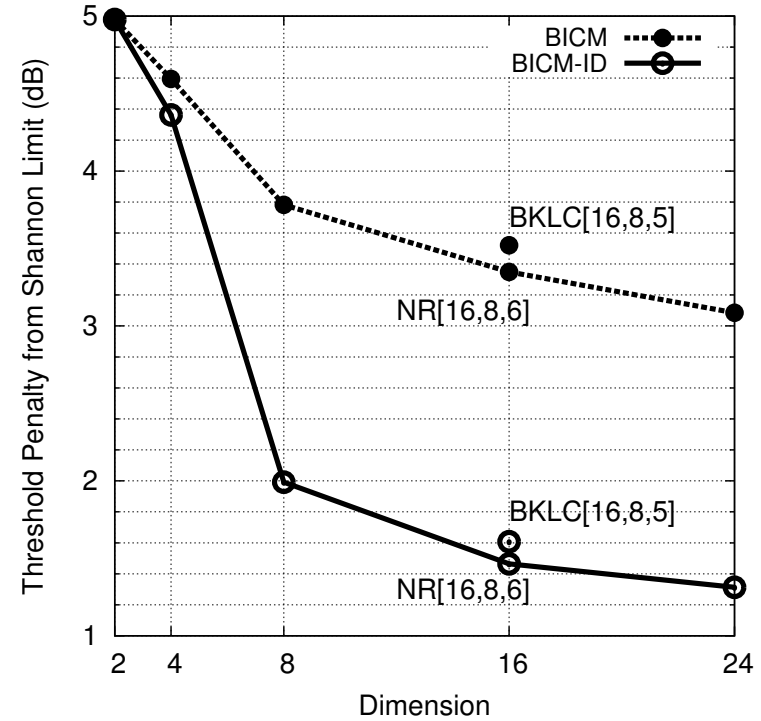

(a) Threshold vs. Dimension

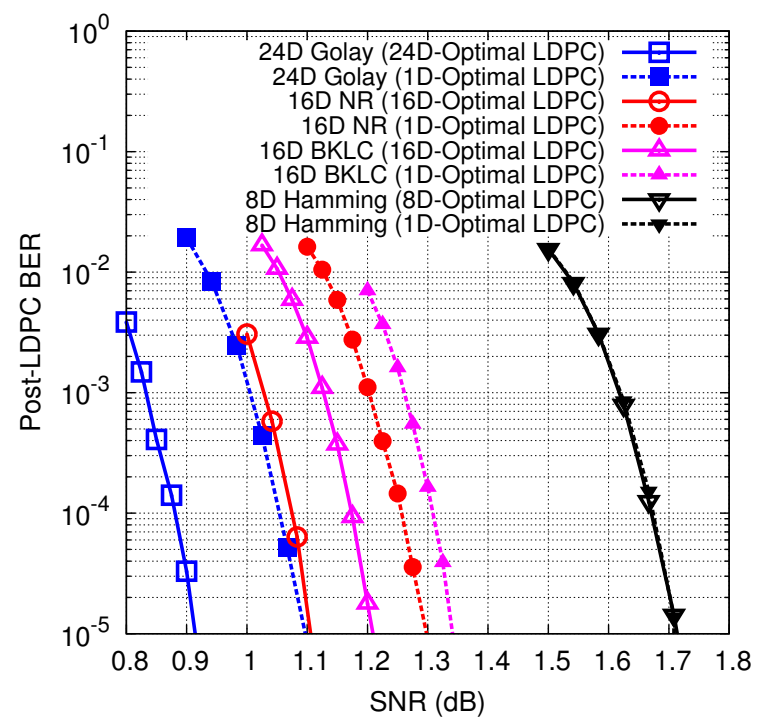

(b) Post-LDPC BER of BICM-ID

Fig. 1: Performance of LDPC-coded HDM for a code rate of 0.9 .

\section{Conclusions}

We proposed LDPC-coded 16D modulation based on the Nordstrom-Robinson (NR) nonlinear code. The proposed HDM approaches performance of 24D modulation based on the extended Golay code within $0.2 \mathrm{~dB}$. It offers $0.6 \mathrm{~dB}$ gain, compared with 8D modulation based on the extended Hamming code, and $0.1 \mathrm{~dB}$ gain, compared with 16D modulation based on the best-known linear code.

\section{References}

1. I.B. Djordjevic, $O F C$ W3J-4 (2014)

2. S.-Y. Chung, G.D. Forney Jr, T.J. Richardson, R. Urbanke, IEEE COMLET 52 (2001)

3. T.J. Richardson, M.A. Shokrollahi, R.L. Urbanke, IEEE TIT 47 2, (2001)

4. S. ten Brink, G. Kramer, A. Ashikhmin, IEEE TCOMM 524 (2004)

5. L. Schmalen, V. Aref, J. Cho, K. Mahdaviani, ECOC Th.1.3.3 (2014)

6. K. Sugihara, Y. Miyata, T. Sugihara, K. Kubo, H. Yoshida, W. Matsumoto, T. Mizuochi, OFC, OM2B.4 (2013)

7. E. Agrell, M. Karlsson, IEEE JLT 27, 5115-5126 (2009)

8. L. Beygi, E. Agrell, J.M. Kahn, M. Karlsson, IEEE JLT 32 2, 333-343 (2013)

9. T. Liu, I.B. Djordjevic, IEEE Photon. J. 64 (2014)

10. D.S. Millar, T. Koike-Akino, S.Ö Arrk, K. Kojima, K. Parsons, OFC M3A.4 (2014)

11. D.S. Millar, T. Koike-Akino, S.Ö Arık, K. Kojima, K. Parsons, T. Yoshida, T. Sugihara, Opt. Exp. 227 (2014)

12. T. Koike-Akino, V. Tarokh, IEEE ICC (2009)

13. A.D. Shiner et al., Opt. Exp. 2217 (2014)

14. A.W. Nordstrom, J.P. Robinson, Information and Control 11, 613-616 (1967)

15. G.D. Forney Jr, N.J.A Sloane, M.D. Trott, In Coding and Quantization: DIMACS/IEEE Workshop 19-26 (1992)

16. Q. Xie, K. Peng, J. Wang, J. Song, J. Wang, Z. Yang, IEEE Trans. Broadcasting 553 (2009)

17. W. Bosma, J. Cannon, C. Playoust, J. Symbolic Comput. 24 235-265 (1997)

18. H. Xiao, A.H. Banihashemi, IEEE COMLET 8 12, 715-717 (2004) 